\title{
Design of a
}

\section{Slot VLPC cryostat}

\section{Cooled by a Cryocooler}

D-ZERO ENGINEERING NOTE \# 3823.115-EN-573

April 22, 2004

Author: Russell A. Rucinski

Project Engineer PPD/MSD/D0 Operations 


\section{Design of a VLPC cryostat, cooled with a commercial cryocooler}

The conceptual design and preliminary engineering calculations have been completed for a two cassette cryostat. This report summarizes the design.

\section{Physical Description, above lid}

See the design sketch of the cryostat. A cryocooler is permanently mounted in the center of a stainless steel, 0.75 inch thick top lid. The cryocooler sits upon a spacer which raises the cooling stage elevations to favorably match the cassette heat intercept elevations. The top lid (32.0" outside diameter) mates to a 24" pipe size flange with o-ring. The 24" pipe size vacuum vessel with end plate has a minimum internal depth of 16 " to give adequate clearance for the depth of the cryocooler and multilayer insulation blankets. Support stand legs elevate the container to a convenient height and allow for placement of the AFE power supply underneath.

Two cassette slots are located on either side of the cryocooler. The slots are positioned parallel to each other, 10.5 " center to center (6 standard cassette slot widths) so that the standard 8 slot AFE backplane can be used. The slot opening through the lid is approximately $1.422 "$ x 16.782 ". A 0.016 " thick titanium (Ti-6Al-4V) envelope with sealing lip is inserted through lid and defines the gas helium boundary that the VLPC cassette resides. The internal dimensions of the titanium envelope are $1.390^{\prime \prime} \mathrm{x} 16.75$ " $\mathrm{x}$ 10.531 " deep. When the cassette is inserted the clearances will be 0.015 " on the long side, 0.063 " on the short side, and 0.032 " at the bottom. The cassette gasket seals against the top lip of the titanium envelope. A soft gasket or thin vacuum sealant tape seals the underside of the titanium envelope to the top surface of the lid. A clamping hold down bar may be necessary to make this vacuum seal.

Gas helium for the cassette space is supplied through a fitting and port that is added to the cassette bulkhead assembly. This is the only modification necessary the standard D-Zero cassette. Evacuation and backfilling and then stagnant positive pressure are achieved through this one port. 


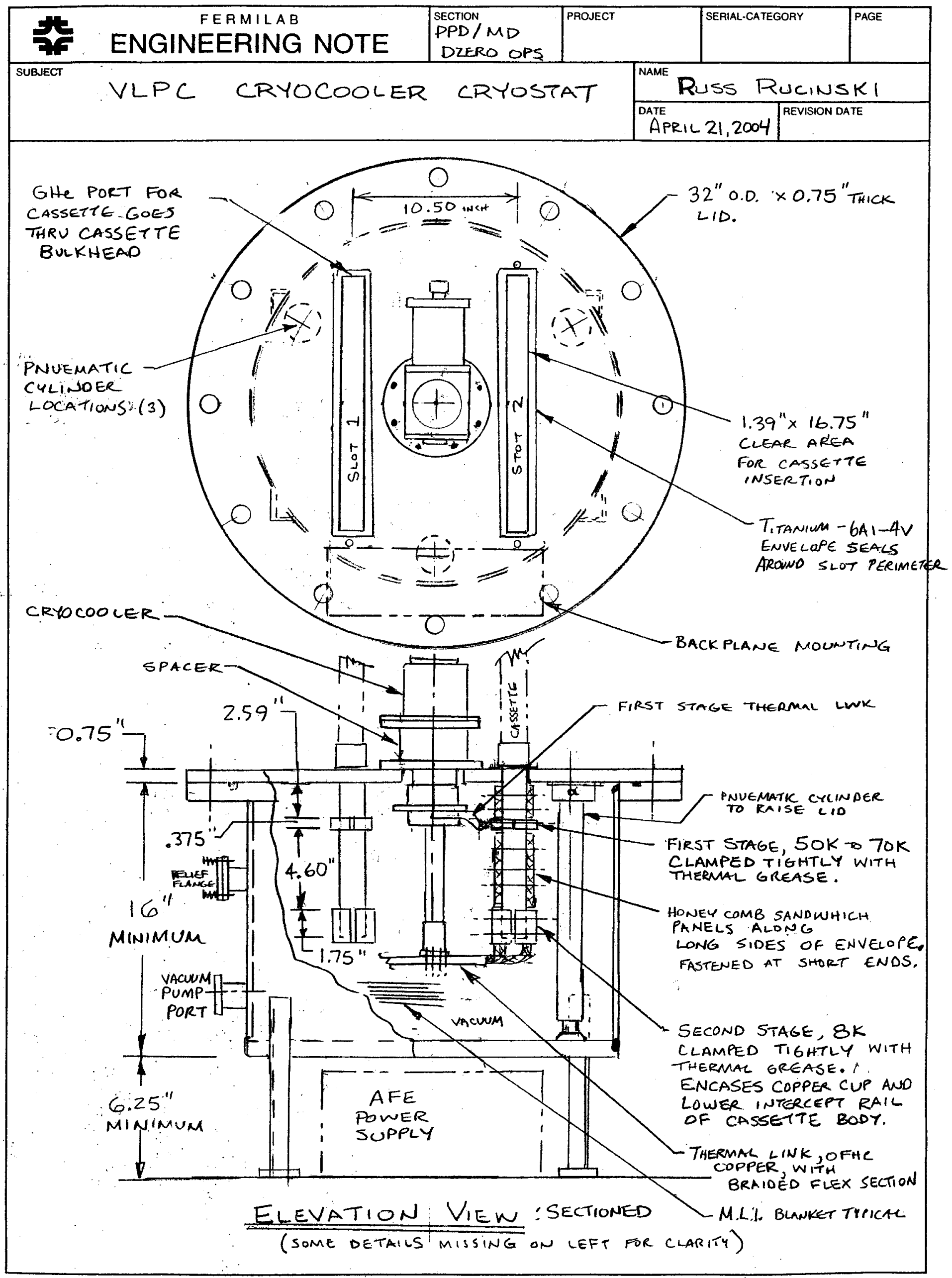


Assembly below the lid

After the titanium envelope/pocket and cassette are mounted to the top lid, the lid is raised so that assembly work can be accomplished below. Three pneumatic cylinders mounted to the lid are pressurized (with air or the gas helium source) to raise the lid. Safety linkages are installed to prevent accidental lowering of the lid while work is being performed.

Honeycomb sandwich panels (http://www.hexcelcomposites.com/default.html or equivalent) are attached to the titanium envelope to support the thin envelope against the pressure differential. A thin thermoplastic mesh placed between the honeycomb panel and titanium envelope helps to insulate the panel so it is not a significant parallel heat path down the wall. There are two panel sets for each cassette. One for the area above the upper intercept and one set for the area below. It is reasonable that a 0.40 " thick panel will suffice.

A high conductance thermal link is made between the first stage of the cryocooler and the uppermost intercept location. This is solid OFHC copper part with a short section of flexible copper braid that connects to bars that clamp solidly to the long sides of the cassette envelope. Apiezon "N" grease (http://www.apiezon.com/grease.htm) is used at assembly to enhance heat transfer at the clamp bar to titanium envelope interface.

A second high conductance thermal connection is made between the second stage of the cryocooler and the bottom of the cassette envelope. The link is mostly solid OFHC copper along with a short flexible braid section in between. The two piece clamping bar encases the bottom 1.75" of the cassette. Thermal grease is used at the interface.

\section{Engineering Details}

Titanium, Ti-6Al-4V, grade 5 was chosen as the envelope material for several reasons. It is compatible with vacuum and cryogenic service. It has about $1 / 2$ the thermal conductivity of 304 stainless steel $(20 \mathrm{~K}$ to $300 \mathrm{~K})$. It's thermal contraction at cryogenic temperatures is about $2 / 3$ that of 304 stainless steel. It is readily available and can be welded and formed as necessary for this design. The yield stress is $128 \mathrm{ksi}$, and the ultimate stress is $138 \mathrm{ksi}$. For design purposes an allowable stress of $1 / 4$ the ultimate, 34.5 $\mathrm{ksi}$ is appropriate. See the website http://asm.matweb.com/search/SpecificMaterial.asp?bassnum=MTP641) for more information about this material.

The maximum allowable working pressure for the cassette space is chosen at 6 psig $=21$ psi absolute. Pressures exceeding this could blow out the cassette gasket. The normal operating pressure of the cassette space is $1.5 \mathrm{psig}$. The envelope wall thickness of 0.016 " was chosen as a practical thickness for fabrication and welding. The envelope is supported against deflection on the outside. Considering an unsupported span of 0.20 " with 21 psid, a wall thickness of 0.005 " would suffice. The 0.016 " thickness is about a factor of three thicker than that necessary for stress or pressure retaining purposes. 
Heat load calculations were done. An existing spreadsheet for cassette heat loads was modified with new inputs for temperature. It was also updated to reflect information known about the production Compunetics flex circuit. Conservative thermal lengths were used. A length of 2.59" (bottom of the lid to the top of the uppermost intercept station) was used along with temperature boundary conditions of $290 \mathrm{~K}$ to $50 \mathrm{~K}$ for the first stage. A length of 4.60" (bottom of the upper intercept to top of lower intercept) was used with $50 \mathrm{~K}$ and $8 \mathrm{~K}$ temperature boundaries. Table 1 gives the heat load results.

The thermal path from the cassette to the heat intercepting bars was estimated. There is some uncertainty in the values used for metal to metal contact resistance especially for the vacuum environment on the outside. Reasonably conservative values were used. To aid in heat transfer, Apiezon " $N$ " grease will be used on the vacuum side of the heat intercepts. The outward pressure differential of 21 psid across the titanium envelope will aid in giving good compressive contact against the thermal bars. Thermal contraction will need to be considered at to ensure the clamping bar fasteners do not loosen after cool down. Table 1 gives the temperature drop estimates for the stations. The temperature drop along the OFHC thermal contact link to the cryocooler is not included. This additional drop should not be significant.

Table 1. Heat Load estimates and Temperature drop estimates

\begin{tabular}{|l|l|l|}
\hline & First Stage (50 Kelvin) & Second Stage (8 K) \\
\hline Cassette & 8.5 Watts & 0.48 Watts \\
\hline Titanium Envelope & 6.9 Watts & 0.20 Watts \\
\hline Miscellaneous & 0.6 Watts & 0.12 Watts \\
\hline \multicolumn{1}{|c|}{ Total heat load $=$} & $\mathbf{1 6}$ Watts per cassette slot & $\mathbf{0 . 8}$ Watts per cassette slot \\
\hline & & \\
\hline$\Delta$ T: Cassette to thermal clamp & $\mathbf{1 6 . 4}$ Kelvin & $\mathbf{0 . 1 8}$ Kelvin \\
\hline
\end{tabular}

The total refrigeration heat load requirements are 32 Watts at the 50 Kelvin stage and 1.6 Watts at the $8 \mathrm{~K}$ stage. The cryocooler that the MICE experiment seems to be standardizing on is the Sumitomo SDRK 415-D cryocooler from Sumitomo Heavy Industries, Ltd., SHI-APD cryogenics, Inc. (http://www.shicryogenics.com/products/). Table 2 shows the cryostat cooling requirement and capacities of two possible cryocoolers. The requirements are well within the capacities offered by many vendors.

Table 2. Heat load requirements and possible cryocooler requirements

\begin{tabular}{|l|l|l|l|}
\hline & Requirement & SDRK 415-D & SRDK-408D \\
\hline $1^{\text {st }}$ Stage $(50-80 \mathrm{~K})$ & 32 Watts & 40 Watts & 40 Watts \\
\hline $2^{\text {nd }}$ Stage $(8 \mathrm{~K})$ & 1.6 Watts & 10 Watts & 6 Watts \\
\hline
\end{tabular}

The actual operating temperature of the first stage was assumed to be 50 Kelvin for the basis of design. A warmer intercept temperature (say up to $80 \mathrm{~K}$ ) will not significantly change the calculations (and may be a better operating regime for the cryocooler). 


\section{Further Considerations}

The gas helium port for the cassette space should come into the volume at the warm end for purposes of steady temperature control. Instead of drilling and tapping a port into the cassette bulkhead, one might consider making a thin titanium sealing flange that contains such a port. The drawback of doing this is that the cassette will sit higher and the thermal length of the titanium skin will be reduced a bit. The titanium envelope will also be more expensive.

Removal of a cassette may be difficult even after releasing the thermal clamps,. In that case, one could surgically cut the titanium skin from the cassette. The titanium skin may not be re-useable for multiple cassettes. Spare titanium envelopes are recommended. 